\title{
THE ABSORPTION OF TRAPPED LINE PHOTONS BY DUST*
}

\author{
Peter Goldreich $\dagger$ aNd John Kwan $\ddagger$ \\ Received 1978 March 2; accepted 1978 June 30
}

\begin{abstract}
We derive the rate at which photons in an optically thick line are absorbed by cold dust. This rate is approximately equal to the dust optical depth to the cloud center times the rate at which the photons escape from the cloud. Our derivation is in response to a recent article by Strel'nitskii in which he incorrectly criticized our previous application of this result to models for the pumping of cosmic masers. Strel'nitskii now agrees that his criticism was unjustified.
\end{abstract}

Subject headings: masers - nebulae: general — radiative transfer

Goldreich and Kwan (1974) (henceforth GK) argue that collisional pumps for cosmic masers are limited by the rate at which infrared line photons are lost either by escape from the cloud or by absorption on cold dust particles. In particular, they assert that the rate of photon absorption by cold dust particles is approximately equal to the dust optical depth to the cloud center times the rate at which photons escape from the cloud. This assertion has been challenged by Strel'nitskii (1977), who claimed that correct allowance for the removal of IR photons by cold dust particles frees the collisional pump mechanism from the thermodynamic difficulty indicated by GK. In this note we provide a proof of our assertion which Strel'nitskii (1978) now agrees is correct.

For simplicity, we consider a model molecule with two scalar levels connected by an allowed dipole transition. The population number densities of the lower and upper levels are $n_{1}$ and $n_{2}$; and the coefficients of spontaneous emission, stimulated emission, and absorption are $A, B_{21}=B_{12}=B$. The equation of transfer in the line is given by

$$
\frac{d I_{v}}{d l}=\frac{h \nu}{4 \pi} \phi(\nu)\left[n_{2} A-\left(n_{1}-n_{2}\right) B I_{v}\right]-n_{d} \sigma_{d} \epsilon_{d}(\nu) I_{v} .
$$

Here $I_{v}$ is the specific intensity along $l, \phi(v)$ is the normalized line profile function (assumed Gaussian), and $n_{d}, \sigma_{d}$, and $\epsilon_{d}(\nu)$ are the number density, the geometrical cross section, and the absorptivity of the dust grains. In writing equation (1), we assume complete frequency redistribution for photons scattered by the molecules. The dust particles are assumed to be cold, so their emission is neglected. Scattering by the dust particles is also ignored since we are interested in infrared photons whose wavelengths are much larger than the dimensions of the dust particles.

\footnotetext{
* Contribution 3046 of the Division of Geological and Planetary Sciences, California Institute of Technology.

$\dagger$ California Institute of Technology.

$\ddagger$ Bell Laboratories, Murray Hill, NJ.
}

The rate equations for the level populations read

$$
\begin{aligned}
\frac{d n_{1}}{d t}=-\frac{d n_{2}}{d t}=n_{2} A & +\left(n_{2}-n_{1}\right) B J \\
+ & {\left[n_{2}-n_{1} \exp \left(-\frac{h v}{k T}\right)\right] C, }
\end{aligned}
$$

where

$$
J=\int J_{v} \phi(v) d \nu=\iint I_{v} \phi(\nu) d \nu \frac{d \Omega}{4 \pi}
$$

is the profile averaged mean intensity, $C$ is the downward collision rate, and $T$ is the kinetic temperature.

The net rate of production of line photons per unit volume due to collisions is

$$
\frac{d n_{\mathrm{ph}}}{d t}=-\left[n_{2}-n_{1} \exp (-h v / k T)\right] C .
$$

In a steady state it follows from equation (2) that

$$
\frac{d n_{\mathrm{ph}}}{d t}=n_{2} A+\left(n_{2}-n_{1}\right) B J
$$

Equation (1) is divided by $h v$ and then integrated over frequency and solid angle, and the result is combined with equation (5). This procedure yields

$$
\frac{d n_{\mathrm{ph}}}{d t}=\iint \frac{1}{h \nu} \frac{d I_{v}}{d l} d \nu d \Omega+\iint n_{d} \sigma_{d} \epsilon_{d}(\nu) \frac{I_{v}}{h \nu} d \nu d \Omega .
$$

Equation (6) may be cast in a more revealing form by noting that

$$
\int \frac{d I_{v}}{d l} d \Omega=\int(\hat{l} \cdot \nabla) I_{v} d \Omega=\int \nabla \cdot\left(I_{v} \hat{l}\right) d \Omega=\nabla \cdot F_{v},
$$

where $\boldsymbol{F}_{v}$ is the photon flux. Thus

$$
\frac{d n_{\mathrm{ph}}}{d t}=\int \frac{1}{h \nu}\left[\boldsymbol{\nabla} \cdot \boldsymbol{F}_{v}+4 \pi n_{d} \sigma_{d} \epsilon_{d}(\nu) J_{v}\right] d v
$$

is seen to be the rate at which photons are lost per unit 
volume due to the divergence of their flux and to their absorption by dust.

The total rate at which photons are lost is obtained by integrating equation (8) over the volume of the cloud. We find

$$
\frac{d N_{\mathrm{ph}}}{d t}=\iint \frac{\boldsymbol{F}_{\nu} \cdot \hat{\boldsymbol{n}} d \nu d A}{h \nu}+4 \pi \iint n_{d} \sigma_{d} \epsilon_{d}(\nu) \frac{J_{v}}{h \nu} d \nu d V,
$$

where $\hat{n}$ is the outward unit normal. For a homogeneous cloud and a line of large optical depth, $J_{v} \approx I_{\nu}$ except within a few optical depths of the surface. Furthermore, $\boldsymbol{F}_{v} \approx \pi I_{v} \hat{\boldsymbol{n}}$ at the cloud's surface. Of course, $I_{v}$ is not exactly the same near the surface of the cloud as in its interior. We ignore this fine distinction and evaluate equation (9) for a spherical cloud of radius $R$ obtaining

$$
\frac{d N_{\mathrm{ph}}}{d t} \simeq 4 \pi^{2} R^{2}\left[1+\frac{4}{3} n_{d} \sigma_{d} \epsilon_{d}(\nu) R\right] \frac{I_{v} \Delta \nu}{h \nu},
$$

where $\Delta \nu$ is the line width. Equation (10) proves the assertion made in GK-namely, the rate at which photons are absorbed by cold dust is approximately equal to the dust optical depth to the cloud center times the rate at which photons escape from the cloud. We note that $d N_{\mathrm{ph}} / d t$ takes on its maximum value if $I_{v}$ is equal to the Planck function $B_{v}(T)$. It is this maximum rate which is quoted in the numerical examples presented by GK.

This research was supported in part by NSF grant AST 76-23281.

\section{REFERENCES}

Goldreich, P., and Kwan, J. 1974, Ap. J., 191, 93 (GK).

Strel'nitskii, V. S. 1977, Astr. Zh., 54, 674 (S).

Strel'nitskii, V. S. 1978, private communication.

Peter Goldreich: Geological and Planetary Sciences, 170-25, California Institute of Technology, Pasadena, CA 91125

JoHN KwAN: Bell Laboratories, 600 Mountain Avenue, Murray Hill, NJ 07974 